\title{
First confirmed ultra-compact dwarf galaxy in the NGC 5044 group
}

\author{
Favio R. Faifer ${ }^{1,2}$, Carlos G. Escudero ${ }^{1,2}$, María C. Scalia ${ }^{1,2}$, Analía V. Smith Castelli2 ${ }^{2,3}$, Mark Norris ${ }^{4}$, \\ María E. De Rossi ${ }^{5,6}$, Juan C. Forte ${ }^{3,7}$, and Sergio A. Cellone $e^{1,2}$ \\ ${ }^{1}$ Facultad de Ciencias Astronómicas y Geofísicas, Universidad Nacional de La Plata, Paseo del Bosque s/n, B1900FWA, La Plata, \\ Argentina \\ e-mail: favio@fcaglp.unlp.edu.ar \\ 2 Instituto de Astrofísica de La Plata (CCT La Plata - CONICET - UNLP), Paseo del Bosque s/n, B1900FWA, La Plata, Argentina \\ 3 Consejo Nacional de Investigaciones Científicas y Técnicas, Godoy Cruz 2290, C1425FQB, CABA, Argentina \\ 4 Jeremiah Horrocks Institute, University of Central Lancashire, Preston, PR1 2HE, UK \\ 5 Universidad de Buenos Aires, Facultad de Ciencias Exactas y Naturales y Ciclo Básico Común, Buenos Aires, Argentina \\ ${ }^{6}$ CONICET-Universidad de Buenos Aires, Instituto de Astronomía y Física del Espacio (IAFE), Argentina \\ 7 Planetario "Galileo Galilei”, Secretaría de Cultura, Av. Sarmiento S/N, 1425 Ciudad Autónoma de Buenos Aires, Argentina
}

Received 24 January 2017 / Accepted 13 February 2017

\begin{abstract}
Context. Ultra-compact dwarfs (UCDs) are stellar systems displaying colours and metallicities between those of globular clusters (GCs) and early-type dwarf galaxies, as well as sizes of $R_{\mathrm{eff}} \lesssim 100 \mathrm{pc}$ and luminosities in the range $-13.5<M_{\mathrm{V}}<-11 \mathrm{mag}$. Although their origin is still subject of debate, the most popular scenarios suggest that they are massive star clusters or the nuclei of tidally stripped dwarf galaxies.

Aims. NGC 5044 is the central massive elliptical galaxy of the NGC 5044 group. Its GC/UCD system is completely unexplored. Methods. In Gemini+GMOS deep images of several fields around NGC 5044 and in spectroscopic multi-object data of one of these fields, we detected an unresolved source with $g^{\prime} \sim 20.6 \mathrm{mag}$, compatible with being an UCD. Its radial velocity was obtained with FXCOR and the penalized pixel-fitting (pPXF) code. To study its stellar population content, we measured the Lick/IDS indices and compared them with predictions of single stellar population models, and we used the full spectral fitting technique.

Results. The spectroscopic analysis of the UCD revealed a radial velocity that agrees with the velocity of the elliptical galaxy NGC 5044. From the Lick/IDS indices, we have obtained a luminosity-weighted age and metallicity of $11.7_{-1.2}^{+1.4} \mathrm{Gyr}$ and $[\mathrm{Z} / \mathrm{H}]=$ $-0.79 \pm 0.04 \mathrm{dex}$, respectively, as well as $[\alpha / \mathrm{Fe}]=0.30 \pm 0.06$. From the full spectral fitting technique, we measured a lower age $(8.52 \mathrm{Gyr})$ and a similar total metallicity $([\mathrm{Z} / \mathrm{H}]=-0.86 \mathrm{dex})$.

Conclusions. Our results indicate that NGC 5044-UCD1 is most likely an extreme GC $\left(M_{V} \sim-12.5\right.$ mag) belonging to the GC system of the elliptical galaxy NGC 5044.
\end{abstract}

Key words. galaxies: elliptical and lenticular, $\mathrm{cD}$ - galaxies: photometry - galaxies: star clusters: general

\section{Introduction}

Ultra-compact dwarfs (UCDs) are stellar systems displaying masses, colours, and metallicities within the range covered by those of globular clusters (GCs) and dwarf galaxies. They show typical effective radii $\left(R_{\text {eff }}\right) \lesssim 100 \mathrm{pc}$ and luminosities in the range $-13.5<M_{\mathrm{V}}<-11 \mathrm{mag}$ (Mieske 2006). It now seems likely that they are a mixed population of massive star clusters and the nuclei of tidally stripped dwarf galaxies (i.e. Hilker 2009; Brodie et al. 2011; Norris \& Kannappan 2011; Pfeffer et al. 2014; Longmore et al. 2014; Zhang et al. 2015). However, it has so far proved difficult to conclusively demonstrate that any individual UCD is of either type (and in particular, that it is of massive star cluster type).

Initially, UCDs were identified in the environments of rich galaxy clusters like Virgo and Fornax. However, more recently, several authors have reported UCDs in groups of galaxies and associated with galaxies in the field (Evstigneeva et al. 2007; Hau et al. 2009; Norris \& Kannappan 2011; Norris et al. 2014). The existence of such objects in low-density environments provides an important insight into the relative frequency of the different formation pathways. However, deep spectroscopy, which gives information about the stellar population content among other properties, is still scarce (Francis et al. 2012; Janz et al. 2016).

We present the spectroscopic confirmation of the first UCD in the vicinity of the massive elliptical galaxy NGC $5044\left(M_{\mathrm{B}}=\right.$ $\left.-21.2 \mathrm{mag}, D=35.7 \pm 5 \mathrm{Mpc}, V_{\mathrm{r}}=2782 \mathrm{~km} \mathrm{~s}^{-1}\right)$, the central object of the NGC 5044 group. This group harbours $\sim 150$ members, most of which are dwarf galaxies (Ferguson \& Sandage 1990). This population represents half the members of the Fornax Cluster. However, its central density and velocity dispersion are higher, while its kinematics and early-type galaxy fraction reveal a mature group (Cellone \& Buzzoni 2005). Although the elliptical galaxy NGC 5044 has been the subject of several studies, its rich GC system $(\sim 5500 \pm 500$ members; Faifer et al., in prep.) remains completely unexplored.

Through the analysis of Gemini+GMOS images and spectra, we have been able to obtain the photometric parameters and the radial velocity of the UCD, as well as information about its stellar population content. The Letter is organized as follows. In Sect. 2 we present our data, in Sect. 3 our analysis and 
Table 1. Log of the GEMINI-GMOS data.

\begin{tabular}{cccc}
\hline \hline Date & Filter/grism & $\begin{array}{c}\text { Exposures } \\
(\mathrm{s})\end{array}$ & $\begin{array}{c}F W H M / \text { resolution } \\
(\operatorname{arcsec} / \AA)\end{array}$ \\
\hline 27 Jan. 2009 & $g^{\prime}$ & $11 \times 450$ & 0.92 \\
& $r^{\prime}$ & $2 \times 350$ & 0.76 \\
& $i^{\prime}$ & $7 \times 350$ & 0.80 \\
26 Feb. 2009 & $g^{\prime}$ & $4 \times 450$ & \\
& $r^{\prime}$ & $2 \times 350$ & \\
22 Mar. 2010 & B600_G5323 & $3 \times 2330$ & 4.61 \\
19 Apr. 2010 & B600_G5323 & $3 \times 2330$ & 4.61 \\
20 Apr. 2010 & B600_G5323 & $2 \times 2330$ & 4.61 \\
\hline
\end{tabular}

results, and in Sect. 4 we discuss the results and we present the conclusions.

\section{Observational data}

With the Gemini Multi-Object Spectrograph (GMOS; Hook et al. 2004) of GEMINI-South, we obtained deep $g^{\prime}, r^{\prime}$, and $i^{\prime}$ images of several fields around NGC 5044 (Program: GS-2009AQ-46). In this Letter we focus on the field that contains the elliptical galaxy. The analysis of the complete photometric dataset will be presented in a forthcoming paper (Faifer et al., in prep.). GMOS consists of three CCDs of $2048 \times 4096$ pixels, separated by gaps of $\sim 2.8$ arcsec, with an unbinned pixel scale of 0.0727 arcsec pixel ${ }^{-1}$. The field of view (FOV) is $5.5^{\prime} \times 5.5^{\prime}$, and the scale for binning $2 \times 2$ is $\sim 0.146$ arcsec pixel $^{-1}$. The images were reduced in the usual way (Escudero et al. 2015).

We have also obtained a GMOS spectroscopic mask of the field containing NGC 5044 with the aim at obtaining radial velocities of a sample of GC candidates (program: GS-2010A-Q56). The instrumental setup included $2 \times 2$ binning and slits of $1^{\prime \prime}$ width, with dithering in the spectral direction in order to fill the CCD gaps. The data were reduced using the IRAF tasks gbias, gsflat, gsreduce, and mgswavelength. The individual spectra were then extracted using apall and were combinded through the task scombine. Finally, an approximate flux calibration was applied based on the flux of the standard star LTT 4816. The final spectrum covers a spectral range of 3750-6780 $\AA$ and displays a resolution of $\sim 4.61 \AA$. The signal-to-noise ratio $(\mathrm{S} / \mathrm{N})$ per $\AA$ of the final spectrum increases from 20 at $4100 \AA$ to 70 at $6000 \AA$. In Table 1 we show the basic information related with all our data.

\section{Analysis and results}

\subsection{Photometry}

We have subtracted the galaxy light from our images using an iterative combination of the background-modelling tool of SExtractor (Bertin \& Arnouts 2010) and a median filtering, following Puzia et al. (2004). SExtractor was also used to perform the detection of GC candidates in the field. At the distance adopted here for NGC 5044, 1 arcsec is equivalent to $170 \mathrm{pc}$. This means that any globular cluster or UCD present in the field will appear as an unresolved source in our images. We have used Daophot to obtain the photometry of the sources detected in the field, and we modelled the psf using $~ 25-30$ well-exposed unresolved objects.

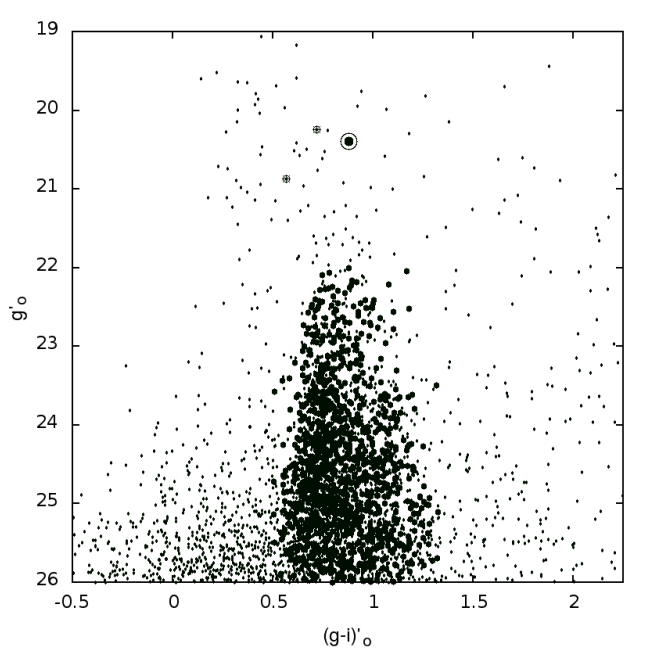

Fig. 1. Colour-magnitude diagram of the unresolved sources detected in the field of NGC 5044 (dots). Small circles depict the objects that match our colour selection for globular clusters. The small circles with a cross inside show two candidates whose radial velocities proved that they are MW stars. The circle surrounded by the ring shows the location of NGC 5044-UCD1 in the diagram.

Figure 1 shows the colour-magnitude diagram for the unresolved sources detected in the field. The magnitudes and colours were calibrated to the Sloan Digital Sky Survey (SDSS) photometric system and corrected for interstellar extinction (Schlafly \& Finkbeiner 2011). Objects with integrated colours in the ranges $0.5<\left(g^{\prime}-i^{\prime}\right)_{0}<1.4,0.35<\left(g^{\prime}-r^{\prime}\right)_{0}<0.85$ and $0.0<\left(r^{\prime}-i^{\prime}\right)_{0}<0.55$ and magnitudes $g_{0}^{\prime}>22$ were considered as GC candidates (Faifer et al. 2011). Three UCD candidates were selected using the same colour ranges, but with $g_{0}^{\prime}<22$, which translates into $M_{V}<-11 \mathrm{mag}$ at the NGC 5044 distance (Mieske et al. 2006).

\subsection{Radial velocity}

A preliminary estimation of the radial velocity $\left(V_{\mathrm{r}}\right)$ of the three UCD candidates was obtained from the spectroscopic data using the task FXCOR from IRAF. As spectral templates, we used the stellar population synthesis models MILES (Vazdekis et al. $2010)$ considering two total metallicities $([\mathrm{Z} / \mathrm{H}]=-1.31$ and $[\mathrm{Z} / \mathrm{H}]=0.00 \mathrm{dex})$, a bimodal IMF with a slope of 1.3 , and ages of 12 Gyr. For two of these objects we have obtained $V_{\mathrm{r}}<400 \mathrm{~km} \mathrm{~s}^{-1}$, meaning that they are Milky Way (MW) stars. For the third object (hereafter, NGC 5044-UCD1) we have obtained $V_{\mathrm{r}} \sim 2900 \mathrm{~km} \mathrm{~s}^{-1}$, similar to the reported systemic velocity of the galaxy NGC $5044\left(2782 \mathrm{~km} \mathrm{~s}^{-1}\right)$, thereby confirming that NGC 5044-UCD1 is a member of this system.

Subsequently, we used the penalized pixel-fitting code pPXF (Cappellari \& Emsellem 2004) in order to mitigate the mismatch template effect and, therefore, to better estimate the $V_{\mathrm{r}}$ of the UCD. We used the SSP MILES library models from Vazdekis et al. (2010), which span a range in age of 0.03-14 Gyr and $-2.27<[\mathrm{Z} / \mathrm{H}]<0.4$ dex. We obtained a radial velocity of $V_{\mathrm{r}} \sim 2945 \mathrm{~km} \mathrm{~s}^{-1}$. This value and the error quoted in Table 2 were obtained as the median from 500 Monte Carlo realizations and the $1 \sigma$ estimation, respectively. Figure 2 shows the integrated spectrum of the UCD together with an example of the fits obtained by pPXF. 
Table 2. Basic information of NGC 5044-UCD1.

\begin{tabular}{lcr}
\hline \hline $\mathrm{RA}(\mathrm{J} 2000)$ & $13: 15: 35.75$ & \\
$\mathrm{Dec}(\mathrm{J} 2000)$ & $-16: 23: 25.33$ & \\
$A_{g}(\mathrm{mag})$ & 0.231 & \\
$A_{r}(\mathrm{mag})$ & 0.160 & \\
$A_{i}(\mathrm{mag})$ & 0.119 & \\
$m_{\mathrm{g}^{\prime}}(\mathrm{mag})$ & 20.62 & \pm 0.01 \\
$\left(g^{\prime}-r^{\prime}\right)(\mathrm{mag})$ & 0.67 & \pm 0.01 \\
$\left(g^{\prime}-i^{\prime}\right)(\mathrm{mag})$ & 0.98 & \pm 0.01 \\
$\left(r^{\prime}-i^{\prime}\right)(\mathrm{mag})$ & 0.31 & \pm 0.01 \\
$V_{\mathrm{r}}\left(\mathrm{km} \mathrm{s} \mathrm{s}^{-1}\right)$ & $2945 \pm 5.00$ & \\
$\mathrm{Age}_{\mathrm{Lick}}(\mathrm{Gyr})$ & 11.7 & \pm 1.4 \\
{$[\mathrm{Z} / \mathrm{H}]_{\mathrm{Lick}}$} & -0.79 & \pm 0.04 \\
{$[\alpha / \mathrm{Fe}]$} & 0.30 & \pm 0.06 \\
$M_{\mathrm{ssp}}\left(M_{\odot}\right)$ & $2.8 \times 10^{7}$ & $\pm 3 \times 10^{6}$ \\
$\mathrm{Age}_{\mathrm{pPXF}}(\mathrm{Gyr})$ & 8.52 & \pm- \\
{$[\mathrm{Z} / \mathrm{H}]_{\mathrm{pPXF}}$} & -0.86 & \pm- \\
$H_{\delta_{A}}(\AA)$ & 1.46 & \pm 0.36 \\
$H_{\delta_{F}}(\AA)$ & 1.53 & \pm 0.24 \\
$G_{4300}(\AA)$ & 3.59 & \pm 0.28 \\
$\mathrm{Fe}_{4383}(\AA)$ & 2.47 & \pm 0.36 \\
$\mathrm{H}_{\beta}(\AA)$ & 2.18 & \pm 0.12 \\
$\mathrm{Fe}_{5015}(\AA)$ & 3.01 & \pm 0.25 \\
$\mathrm{Mgb}(\AA)$ & 2.35 & \pm 0.11 \\
$\mathrm{Fe}_{5270}(\AA)$ & 1.68 & \pm 0.13 \\
$\mathrm{Fe}_{5335}(\AA)$ & 1.69 & \pm 0.14 \\
$\mathrm{Fe}_{5406}(\AA)$ & 0.74 & \pm 0.11 \\
{$[\mathrm{MgFe}]^{\prime}(\AA)$} & 1.99 & \pm 0.11 \\
$\langle\mathrm{Fe}\rangle(\AA)$ & 1.69 & \pm 0.10
\end{tabular}

Notes. Apparent magnitudes and colours are not extinction or reddening corrected.

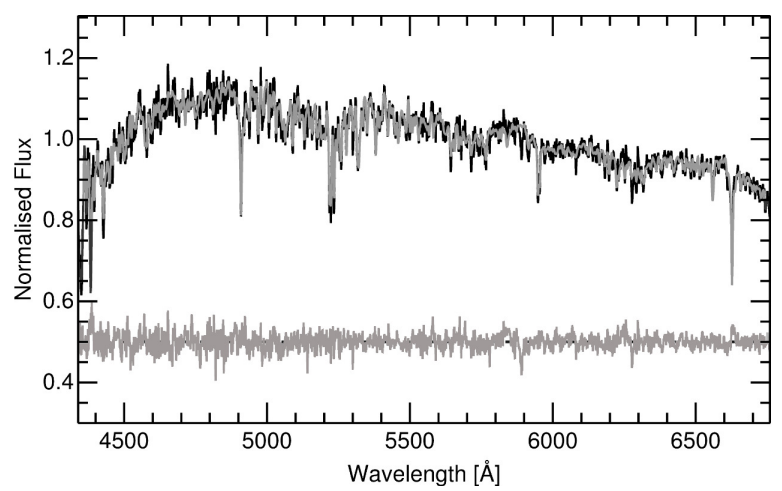

Fig. 2. Integrated spectrum of NGC 5044-UCD1 (black) and one of the kinematics fits obtained by pPXF (grey). We also show the residuals of the fit offset by 0.5 , for clarity.

\subsection{Age and metalicity from Lick indices and SSP models}

We have obtained the Lick indices from the GMOS spectra following Norris et al.; Norris et al. (2008; 2015; Table 2). Uncertainties for the indices were obtained from 500 Monte Carlo simulations based on a combination of errors introduced by photon noise (both target and sky) and errors in the measured redshift of the spectra (essentially negligible here). The age, metallicity, and $\alpha$-elements ratio of NGC 5044-UCD1 were obtained through the $\chi^{2}$ minimization method of Proctor \& Sansom (2002) and Proctor et al. (2004). We have used a smooth grid in age, $[\alpha / \mathrm{Fe}]$, and $[\mathrm{Z} / \mathrm{H}]$ by interpolating the single stellar population (SSP)

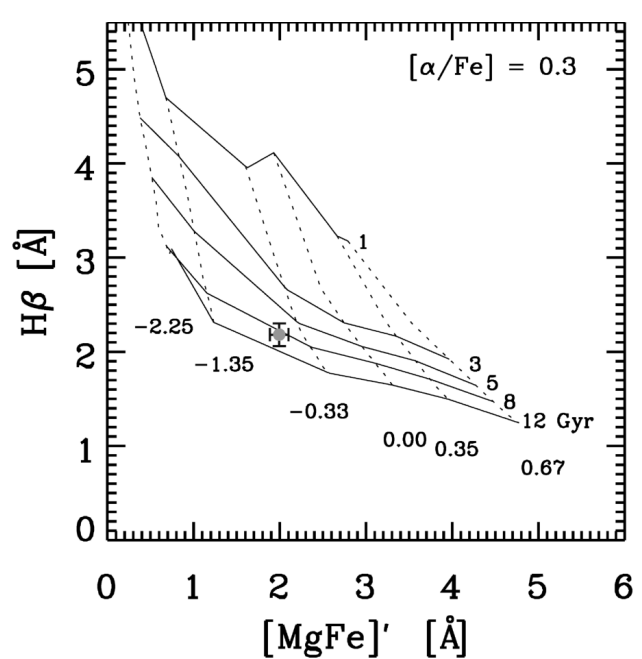

Fig. 3. Diagnostic diagram $\mathrm{H}_{\beta}$ vs. $[\mathrm{MgFe}]$. We also overplot the models of Thomas et al. $(2003,2004)$ with $[\alpha / \mathrm{Fe}]=0.3$. NGC 5044UCD1 (filled circle with error bars) is an ancient object with subsolar metallicity.

models of Thomas et al. (2003, 2004). As in similar works (e.g. Norris et al. 2006), the indices $\mathrm{H}_{\delta A}, \mathrm{H}_{\delta F}, \mathrm{H} \gamma A$ G4300, Fe4383, $\mathrm{H} \beta, \mathrm{Fe} 5015, \mathrm{Mgb}, \mathrm{Fe} 5270, \mathrm{Fe} 5335$, and Fe5406 gave the most reliable results. The analysis provides an age of $11.7_{-1.2}^{+1.4} \mathrm{Gyr}$, a total metallicity of $[\mathrm{Z} / \mathrm{H}]=-0.79 \pm 0.04$ dex, and an $\alpha$-elements ratio of $[\alpha / \mathrm{Fe}]=0.30 \pm 0.06$. From these values and using the $M / L$ ratios given by Maraston (2005) for a Kroupa IMF $(M / L=$ $2.9 \pm 0.3)$, we infer a total stellar mass of $2.8( \pm 0.3) \times 10^{7} M_{\odot}$. In Fig. 3 we show the diagnostic diagram $H_{\beta}$ vs. $[\mathrm{Mg} \mathrm{Fe}]$, where $[\mathrm{MgFe}]^{\prime}=[\mathrm{Mgb} \times(0.72 \times \mathrm{Fe} 5270+0.28 \times \mathrm{Fe} 5335)]^{0.5}$, overplotting the models of Thomas et al. $(2003,2004)$ with $[\alpha / \mathrm{Fe}]=0.3$, according to the value previously estimated.

\subsection{Star formation history}

Through the full spectral fitting technique implemented within the pPXF code, we studied the star formation history (SFH) of NGC 5044-UCD1 and obtained new estimates of the age and metallicity of this object in a way that is independent of that presented in Sect. 3.3. We applied the regularization method to obtain a smooth solution of the best fit linear combination of SSP models to the UCD spectrum (McDermid et al. 2015). We used the same library of models presented in Sect. 3.2, adopting an $\alpha$-element ratio of $[\alpha / \mathrm{Fe}]=0.4$, which is close to the value obtained in the Lick index analysis.

We performed several pPXF runs considering different spectral ranges of the UCD spectrum. In all the cases, we achieved a smooth solution that included a main contribution of models around a mean age of $8.52 \mathrm{Gyr}$ and a mean metalicity of $[\mathrm{Z} / \mathrm{H}]=-0.86 \mathrm{dex}$ (Fig. 4). In some of the tests, which include the regions of the highest $\mathrm{S} / \mathrm{N}(\lambda>4500 \AA)$, we also obtained a secondary contribution of models with higher metallicities $([\mathrm{Z} / \mathrm{H}]>-0.25$ dex $)$. In Fig. 4 we show one of the tests with both contributions. To check this result, we built a synthetic spectrum combining two SSP models with the ages and metallicities obtained from our analysis for the two components. We added to this spectrum a noise level similar to that found in the observed spectrum, and we ran pPFX. We recovered the original two components, but a third spurious subpopulation was also obtained. To confirm this result, we would need a spectrum displaying a higher $\mathrm{S} / \mathrm{N}$ in the whole spectral range. 


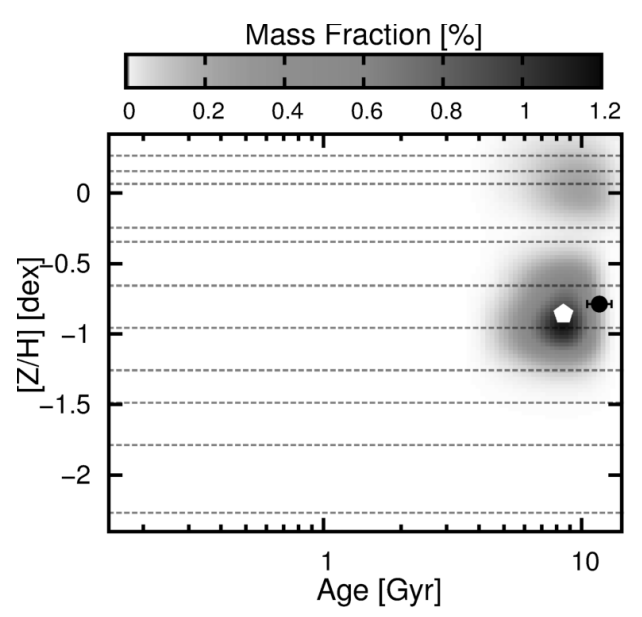

Fig. 4. Star formation history of NGC 5044-UCD1 obtained using pPXF. The different grey-scale regions indicate the weight of each SSP model, which is equivalent to the zero-age mass distribution of the $\mathrm{SFH}$. The dashed lines indicate the $[\mathrm{Z} / \mathrm{H}]$ of each available model. The black filled circle depicts the luminosity-weighted SSP age and metallicity of this object as measured using absorption line strength indices (Sect. 3.3). The white pentagon shows the location of the massweighted age and metallicity derived from the full spectral fitting technique, and considering only models with $[\mathrm{Z} / \mathrm{H}]<-0.25$ dex.

\section{Discussion and conclusions}

From the photometric and spectroscopic analysis of a deep field taken with Gemini+GMOS, we have been able to detect and confirm the first UCD in the NGC 5044 group. Its radial velocity and angular proximity $(2.83 \mathrm{arcmin})$ indicate that this object is associated with the massive early-type galaxy NGC 5044. At the distance adopted in this work for this galaxy, this UCD appears to be an unresolved object, and it has an absolute magnitude of $M_{V}=-12.5 \mathrm{mag}$. This luminosity is well above the usual upper cut for "classical" globular clusters (Faifer et al. 2011).

We studied its stellar population properties using two methods: the analysis of Lick/IDS indices, and the application of the full spectral fitting technique. We find a discrepancy in the age determined by the two methods. From the comparison of the Lick/IDS values with SSP models, we have obtained an age of $11.7_{-1.2}^{+1.4} \mathrm{Gyr}$, which is significantly older than the value of $8.5 \mathrm{Gyr}$ obtained with the full spectral fitting technique, although the uncertainties on the full spectral fitting age are difficult to quantify. Similar discrepancies have been reported for UCDs before (e.g. Francis et al. 2012). However, in contrast to most of the objects presented by Francis and collaborators, our metallicity values are consistent regardless of the method used $([\mathrm{Z} / \mathrm{H}]=-0.79 \pm 0.04 \operatorname{dex}$ and $[\mathrm{Z} / \mathrm{H}]=-0.86 \mathrm{dex})$. It is notable that the metallicity of NGC 5044-UCD1 is within the range displayed by the UCDs detected in Virgo and Fornax, but considerably lower than that of the confirmed stripped nuclei presented by Janz et al. (2016).

In common with most globular clusters (Puzia et al. 2005; Norris et al. 2008), NGC 5044-UCD1 presents a supersolar $\alpha$-element abundance of $[\alpha / \mathrm{Fe}]=0.30$, suggesting rapid star formation. The SFH analysis suggests the presence of a secondary metal-rich stellar population, of an age indistinguishable from the main population. If confirmed by higher $\mathrm{S} / \mathrm{N}$ data, this result could be associated with the "blue tilt" phenomena of massive GCs, whereby the most massive low-metallicity GCs are found to be progressively redder. This has been interpreted as being due to self-enrichment, where the most massive GCs are able to form additional generations of stars enriched in metals from the ejecta of the initial population (Strader \& Smith 2008).

According to Francis et al. (2012), the UCDs of Virgo and Fornax do not display properties similar to those of the nuclei of present-day nucleated dwarf galaxies. This picture seems to be in agreement with the location that our UCD would have in Fig. 13 of Norris \& Kannappan (2011). In that figure, the absolute visual magnitude of our object $\left(M_{V}=-12.5 \mathrm{mag}\right)$ and the total number of GCs of NGC $5044(\sim 5500 \pm 500$, Faifer et al. in prep.) would agree with the general trend defined by the UCDs that are more likely the brightest tail of the GC population. In this sense, from a Monte Carlo experiment using the luminosity function of the NGC 5044 GC system, we have obtained that in $30 \%$ of the realizations we have $1-3$ GCs brighter than $M_{V}=-12.5 \mathrm{mag}$. Therefore, all the lines of evidence would point toward NGC 5044-UCD1 being an unusually massive GC of the NGC 5044 system.

Acknowledgements. Based on observations obtained at the Gemini Observatory (Programs GS-2009A-Q-46 and GS-2010A-Q-56), which is operated by the Association of Universities for Research in Astronomy, Inc., under a cooperative agreement with the NSF on behalf of the Gemini partnership: the National Science Foundation (United States), the National Research Council (Canada), CONICYT (Chile), the Australian Research Council (Australia), Ministério da Ciência, Tecnologia e Inovação (Brazil) and Ministerio de Ciencia, Tecnología e Innovación Productiva (Argentina). This research has made use of the NASA/IPAC Extragalactic Database (NED) which is operated by the Jet Propulsion Laboratory, California Institute of Technology, under contract with the National Aeronautics and Space Administration. This work was funded with grants from Consejo Nacional de Investigaciones Científicas y Técnicas and Universidad Nacional de La Plata (Argentina).

\section{References}

Bertin, E., \& Arnouts, S. 2010, Astrophysics Source Code Library [record ascl: 1010.064]

Brodie, J. P., Romanowsky, A. J., Strader, J., \& Forbes, D. A. 2011, AJ, 142, 199 Cappellari, M., \& Emsellem, E. 2004, PASP, 116, 138

Cellone, S. A., \& Buzzoni, A. 2005, MNRAS, 356, 41

Escudero, C. G., Faifer, F. R., Bassino, L. P., Calderón, J. P., \& Caso, J. P. 2015, MNRAS, 449, 612

Evstigneeva, E. A., Drinkwater, M. J., Jurek, R., et al. 2007, MNRAS, 378, 1036 Faifer, F. R., Forte, J. C., Norris, M. A., et al. 2011, MNRAS, 416, 155 Ferguson, H. C., \& Sandage, A. 1990, AJ, 100, 1

Francis, K. J., Drinkwater, M. J., Chilingarian, I. V., Bolt, A. M., \& Firth, P. 2012, MNRAS, 425, 325

Hau, G. K. T., Spitler, L. R., Forbes, D. A., et al. 2009, MNRAS, 394, L97 Hilker, M. 2009, in Reviews in Modern Astronomy, vol. 21, ed. S. Röser (Wiley), 199

Hook, I. M., Jørgensen, I., Allington-Smith, J. R., et al. 2004, PASP, 116, 425 Janz, J., Norris, M. A., Forbes, D. A., et al. 2016, MNRAS, 456, 617 Longmore, S. N., Kruijssen, J. M. D., Bastian, N., et al. 2014, Protostars and Planets VI, 291

Maraston, C. 2005, MNRAS, 362, 799

McDermid, R. M., Alatalo, K., Blitz, L., et al. 2015, MNRAS, 448, 3484

Mieske, S. 2006, in Rev. Mex. Astron. Astrofis. Conf. Ser., 26, 194

Mieske, S., Hilker, M., Infante, L., \& Jordán, A. 2006, AJ, 131, 2442

Norris, M. A., \& Kannappan, S. J. 2011, MNRAS, 414, 739

Norris, M. A., Sharples, R. M., \& Kuntschner, H. 2006, MNRAS, 367, 815

Norris, M. A., Sharples, R. M., Bridges, T., et al. 2008, MNRAS, 385, 40

Norris, M. A., Kannappan, S. J., Forbes, D. A., et al. 2014, MNRAS, 443, 1151

Norris, M. A., Escudero, C. G., Faifer, F. R., et al. 2015, MNRAS, 451, 3615

Pfeffer, J., Griffen, B. F., Baumgardt, H., \& Hilker, M. 2014, MNRAS, 444, 3670

Proctor, R. N., \& Sansom, A. E. 2002, MNRAS, 333, 517

Proctor, R. N., Forbes, D. A., \& Beasley, M. A. 2004, MNRAS, 355, 1327

Puzia, T. H., Kissler-Patig, M., Thomas, D., et al. 2004, A\&A, 415, 123

Puzia, T. H., Kissler-Patig, M., Thomas, D., et al. 2005, A\&A, 439, 997

Schlafly, E. F., \& Finkbeiner, D. P. 2011, ApJ, 737, 103

Strader, J., \& Smith, G. H. 2008, AJ, 136, 1828

Thomas, D., Maraston, C., \& Bender, R. 2003, MNRAS, 339, 897

Thomas, D., Maraston, C., \& Korn, A. 2004, MNRAS, 351, L19

Vazdekis, A., Sánchez-Blázquez, P., Falcón-Barroso, J., et al. 2010, MNRAS, 404, 1639

Zhang, H.-X., Peng, E. W., Côté, P., et al. 2015, ApJ, 802, 30 